\title{
Rückzug einer Volksinitiative nach aufgehobener Volksabstimmung
}

Besprechung des Urteils des Bundesgerichts 1C_105/2020, 1C_129/2020 vom 7. Oktober 2020 i.S. Rückzug der Volksinitiative «Für Ehe und Familie - gegen die Heiratsstrafe» (zur Publikation bestimmt)

Im Nachgang zur Aufhebung der Volksabstimmung über die Volksinitiative «Für Ehe und Familie - gegen die Heiratsstrafe» (BGE145 I 207) hatte das Bundesgericht zu entscheiden, ob das Initiativkomitee erneut Gelegenheit erhalten sollte, seine Initiative zurückzuziehen, oder ob über die Initiative zwingend wieder abzustimmen sei. Mit einer weitgehend überzeugenden Begründung weist das Bundesgericht die Beschwerde ab und spricht sich dafür aus, dem Initiativkomitee den Rückzug der Initiative zu gestatten. Dass das Gericht die Beschwerde zulässt, ist mit Blick auf die Rechtsweggarantie und den breiten Anwendungsbereich der Beschwerde in Stimmrechtssachen zu begrüssen. Das Urteil lässt dabei aber eine Reihe von prozessualen Fragen ungeklärt, was aus Sicht der Praxis der Stimmrechtsbeschwerde zu bedauern ist. So hätte das Bundesgericht zumindest klarstellen sollen, dass die Rückzugserklärung des Initiativkomitees selbst Anfechtungsobjekt der Beschwerde bildet und nicht die Bekanntmachung des Rückzugs durch die Bundeskanzlei.

I. Weiterungen einer Premiere

Zitiervorschlag:

GORAN SEFEROVIC, Rückzug einer Volksinitiative nach aufgehobener Volksabstimmung, sui generis 2021, S. 37

PD Dr. iur. Goran Seferovic (goran.seferovic@zhaw.ch) ist stellvertretender Leiter des Zentrums für öffentliches Wirtschaftsrecht an der ZHAW School of Management and Law in Winterthur, Privatdozent an der Universität Zürich sowie Rechtsanwalt bei Abegg Anwälte und Konsulenten in Zürich.

URL: sui-generis.ch/166

DOI: https://doi.org/10.21257/sg.166

Dieses Werk ist lizenziert unter einer Creative Commons Namensnennung - Weitergabe unter gleichen Bedingungen 4.0 International Lizenz. 


\section{Weiterungen einer Premiere}

1 ImJahre 2016 feierte die Schweiz das 125-Jahres-Jubiläum der Volksinitiative auf Teilrevision der Bundesverfassung. ${ }^{1}$ Es dauerte weitere drei Jahre, bis das Bundesgericht zum ersten Mal überhaupt eine eidgenössische Volksabstimmung aufhob. Der Fall betraf die Volksabstimmung über die von der CVP Schweiz lancierte Volksinitiative «Für Ehe und Familie - gegen die Heiratsstrafe», welche mit 50.8\% der Volksstimmen abgelehnt wurde, während 16,5Standesstimmen für die Initiative resultierten. ${ }^{2}$ Das höchste Gericht annullierte die Volksabstimmung aufgrund eines Verstosses gegen die Abstimmungsfreiheit von Art. 34 Abs. 2 BV. ${ }^{3}$ Dies in erster Linie deshalb, weil die Behörden die Stimmberechtigten im Vorfeld der Abstimmung mangelhaft über die Anzahl steuerlich ungleich behandelter Ehepaare informiert hatten. ${ }^{4}$

2 In jenem Urteil forderte das Bundesgericht den Bundesrat auf, seinen Erwahrungsbeschluss über die Volksabstimmung von Amtes wegen aufzuheben. ${ }^{5}$ Was danach geschehen sollte, liess das Gericht offen. Angesichts seiner Singularität regelt auch das Bundesrecht einen solchen Fall nicht ausdrücklich. ${ }^{6}$

3 Der Bundesrat hob seinen Erwahrungsbeschluss am 21.Juni 2019 auf.7 Den Initianten war es inzwischen jedoch nicht mehr wohl mit ihrer Initiative, ${ }^{8}$ da diese neben der steuerlichen Gleichbehandlung von Ehepaaren auch eine Definition der Ehe als Lebensgemeinschaft von Mann und Frau in die Bundesverfassung einfügen wollte. ${ }^{9}$ Das Initiativkomitee zog die Initiative daher mit Erklärung bei der Bundeskanzlei zurück. Die Bundeskanzlei publizierte diese zusammen mit der Ankündigung, wonach der Bundesrat deshalb keine Volksabstimmung über die Initiative ansetzen werde..$^{10}$

1 Vgl. Bundesbeschluss vom 29. Juli 1891 betreffend die Erwahrung der Volksabstimmung vom 5. Juli 1891 über die Revision des dritten Abschnittes der Bundesverfassung vom 29. Mai 1874, handelnd von der Revision der Bundesverfassung (AS XII161).

2 Bundesratsbeschluss vom 19. April 2016 über das Ergebnis der Volksabstimmung vom 28. Februar 2016 (BBl 2016 3715).

3 Bundesverfassung der Schweizerischen Eidgenossenschaft vom 18. April 1999 (BV; SR101).

4 BGE145I207 insb. E. 3 und 4.

5 BGE145I207 E.4.2.

6 Vgl. Urteil des Bundesgerichts 1C_105/2020, 1C 129/2020 vom 7. Oktober 2020 E.3.3.

7 Bundesratsbeschluss vom 21. Juni 2019 über die Aufhebung der Er wahrung des Ergebnisses der Volksabstimmung vom 28. Februar 2016 über die Volksinitiative «Für Ehe und Familie - gegen die Heiratsstrafe» (BBl 20194599).

8 Vgl. etwa NZZ online vom 12. Februar 2020 (CVP zieht Initiative zur Abschaffung der Heiratsstrafe zurück).

9 Art.14 Abs. 2 (neu), Bundesbeschluss Entwurf über die Volksinitiative «Für Ehe und Familie - gegen die Heiratsstrafe», Abstimmungsvorlage (BBl 20138541).

10 Eidgenössische Volksinitiative «Für Ehe und Familie - gegen die Heiratsstrafe» Rückzug (BBl 20201284).
Mit diesem Vorgehen waren die Beschwerdeführer nicht 4 einverstanden, weshalb sie sich mit Beschwerde in öffentlich-rechtlichen Angelegenheiten ans Bundesgericht wandten.

\section{Unklarheiten des Rechtsweges}

Die Besonderheit des Falles zeigte sich bereits bei der 5 Frage des Rechtsweges. Die beiden Prozesse, welche das Bundesgericht schliesslich vereinigte, richteten sich gegen unterschiedliche Anfechtungsobjekte. Der eine Beschwerdeführer führte direkt Beschwerde am Bundesgericht gegen die Anzeige der Bundeskanzlei, wonach das Initiativkomitee die Initiative zurückgezogen habe und der Bundesrat deshalb keine Abstimmung ansetzen werde. ${ }^{11}$ Die anderen Beschwerdeführer fochten mit ihrer Beschwerde hingegen den Rückzugsbeschluss des Initiativkomitees an und hielten sich dazu an den Beschwerdeweg, wie ihn das Bundesrecht für eidgenössische Abstimmungssachen in Art. 77 und 80 BPR ${ }^{12}$ vorsieht. ${ }^{13}$ Unbesehen der gerügten Unregelmässigkeiten ist nach diesen Bestimmungen zuerst Beschwerde bei der Kantonsregierung zu führen und erst in zweiter Instanz am Bundesgericht.

Noch bevor das Bundesgericht sich mit dem einzuschla- 6 genden Rechtsweg befassen konnte, hatte es aber die Frage zu klären, ob gegen einen Rückzug einer Volksinitiative überhaupt ein Rechtsmittel bestehen sollte. Die Bundeskanzlei stellte sich auf den Standpunkt, das BPR sehe kein Rechtsmittel gegen den Rückzug einer Volksinitiative vor, räumte angesichts der bisherigen Praxis des Bundesgerichts aber gleichwohl ein, dass das höchste Gericht im Bereich der politischen Rechte schon verschiedentlich Rechtsschutz direkt gestützt auf Art. 29a i.V.m. Art. 34 BV gewährt habe. ${ }^{14}$

Auch nach der Auffassung des Bundesgerichts können 7 Stimmberechtigte weder mit der Abstimmungsbeschwerde (Art. 77 Abs. 1 lit. b BPR) noch mit der Stimmrechtsbeschwerde (Art. 77 Abs. 1 lit. a BPR) den Rückzug einer Volksinitiative anfechten. ${ }^{15}$ Ebenso lasse sich auch eine

11 Urteil des Bundesgerichts 1C_105/2020,1C_129/2020 vom 7. Oktober 2020 E. 2.1.

12 Bundesgesetz über die politischen Rechte vom 17. Dezember 1976 (BPR; SR 161.1).

13 Urteil des Bundesgerichts 1C 105/2020, 1C 129/2020 vom 7. Oktober 2020 E.2.1.

14 Urteil des Bundesgerichts 1C_105/2020,1C_129/2020 vom 7. Oktober 2020 E. 2.1.

15 Urteil des Bundesgerichts 1C_105/2020,1C_129/2020 vom 7. Oktober 2020 E. 2.2. Immerhin hält das Bundesgericht in einer Klammerbemerkung fest, dass der Anspruch auf freie Willensbildung selbstverständlich auch bereits in der Phase der Unterschriftensammlung für eine Initiative oder ein Referendum zum Tragen kommt und nicht erst nach Festsetzung der Volksabstimmung. 
direkte Beschwerde gegen die Erklärung der Bundeskanzlei nicht direkt auf das BPR stützen. Art. 80 Abs. 2 und 3 BPR sehen zwar Beschwerden gegen Verfügungen der Bundeskanzlei vor, jedoch nicht im Falle der Publikation einer Rückzugserklärung eines Initiativkomitees.

8 Dies schliesse aber den Rechtsschutz im Bereich der politischen Rechte, welchen das Bundesgericht aus der Rechtsweggarantie von Art. 29a BV ableite, nicht grundsätzlich aus. Im Gegenteil hätte sich eine solche Ausnahme von der Rechtsweggarantie nach Art. 29a Satz 2 BV hinreichend klar aus dem Gesetz zu ergeben. Da das BPR diesen Rechtsschutz aber gerade nicht ausschliesse, sei dieses verfassungskonform «auszulegen und anzuwenden». ${ }^{16}$ Das Bundesgericht verweist dazu auf seinen Entscheid über die eidgenössische Volksabstimmung zur Unternehmenssteuerreform II, in welchem das Gericht sich für zuständig erklärt hatte, eidgenössische Abstimmungen nachträglich zu überprüfen. ${ }^{17}$

9 Das Bundesgericht folgt der Bundeskanzlei auch bei der Frage, ob eine Beschwerde in Analogie zu Art. 77 BPR zuerst bei der zuständigen Kantonsregierung oder analog zu Art. 80 Abs. 2 und 3 BPR direkt am Bundesgericht einzureichen sei. Mit dem eher rechtspolitischen Argument, es sei «kaum nachvollziehbar», wenn eine Kantonsregierung über den Rückzug einer eidgenössischen Volksinitiative entscheiden müsse, spricht sich das Gericht dafür aus, die direkte Beschwerde ans Bundesgericht zuzulassen. ${ }^{18}$ Nichtsdestotrotz behandelt das Gericht mit Hinweis auf die unklare Rechtslage aber auch die Beschwerde gegen den Entscheid des Regierungsrates des Kantons Bern. ${ }^{19}$ Es nimmt beide Beschwerden als Stimmrechtsbeschwerden i.S.v. Art. 82 lit. c BGG an. ${ }^{20} \mathrm{Im}$ Gegensatz zu den natürlichen Personen verneint das Gericht jedoch die Legitimation des Vereins Human Life International (HLI-Schweiz) und bestätigt damit die zuletzt wieder enger gefasste Legitimation juristischer Personen zur Stimmrechtsbeschwerde. ${ }^{21}$

10 Wenngleich das Eintreten auf beide Beschwerden aus Gründen der Rechtssicherheit zu begrüssen ist, so lässt das Bundesgericht mit Verweis auf den Verfahrensausgang leider offen, welches denn das Anfechtungsobjekt

16 Urteil des Bundesgerichts 1C_105/2020, 1C_129/2020 vom 7. Oktober 2020 E. 2.3 .

17 BGE138I61 E.4.8. Vgl. dazu GIOvANNI BIAGGINI, Eine verzwickte Angelegenheit: Die nachträgliche Überprüfung der Regularität einer eidgenössischen Volksabstimmung, ZBl 2012, S. $429 \mathrm{ff}$.

18 Urteil des Bundesgerichts 1C_105/2020, 1C 129/2020 vom 7. Oktober 2020 E. 2.4

19 Urteil des Bundesgerichts 1C 105/2020, 1C 129/2020 vom 7. Oktober 2020 E. 2.4

20 Urteil des Bundesgerichts 1 C 105/2020,1C 129/2020 vom 7. Oktober 2020 E. 2.6

21 Urteil des Bundesgerichts 1C_105/2020,1C_129/2020 vom 7. Oktober 2020 E. 2.5 . der beiden Beschwerden darstellt. Die Beschwerdeführer richteten ihre Beschwerden im einen Fall gegen den Rückzugsentscheid des Initiativkomitees (1C_129/2020), im anderen Fall gegen die Anzeige der Bundeskanzlei über den Rückzug der Initiative, welche sie als Feststellungsverfügung qualifizierten (1C_105/2020). Das Rückzugsrecht ist das Recht, einen gestellten Antrag zurückzuziehen, über welchen sonst rechtsverbindlich zu beschliessen wäre. ${ }^{22}$ Es ist somit einseitig am Initiativkomitee, durch die Rückzugserklärung die entsprechenden Rechtswirkungen auszulösen, womit kein Raum für eine rechtsgestaltende Verfügung durch die Bundeskanzlei verbleibt. Art. 73 Abs. 2 BPR sieht denn auch vor, dass die Bundeskanzlei das Initiativkomitee vor Ansetzung der Volksabstimmung durch den Bundesrat einlädt, seinen Entscheid über einen Rückzug bekanntzugeben. Die Anzeige der Bundeskanzlei kann aus diesem Grund auch keine Feststellungsverfügung darstellen, da kein schutzwürdiges Interesse an einer solchen bestehen kann. ${ }^{23}$ Die Anzeige der Bundeskanzlei klärt lediglich die tatsächlichen Verhältnisse, indem sie öffentlich bekanntmacht, dass das Initiativkomitee sein Rückzugsrecht ausgeübt hat. ${ }^{24}$ Anfechtungsobjekt stellt daher die Rückzugserklärung des Initiativkomitees selbst dar und nicht die Bekanntmachung dieses Rückzugsentscheids durch die Bundeskanzlei. ${ }^{25}$

\section{Wiederaufleben des Rückzugs- rechts?}

Das Bundesgericht hatte im Kern eine ausgesprochen 11 dogmatische Frage zu entscheiden: Welche Rechtswirkungen hat die Aufhebung einer Volksabstimmung über eine Volksinitiative in der Form des ausgearbeiteten Entwurfs auf Bundesebene darüber hinaus, als dass die Abstimmung ex nunc aufgehoben wird?

\section{Standpunkt der Beschwerdeführer}

Die Beschwerdeführer argumentierten für ein sehr enges 12 Verständnis des vorangehenden Urteils des Bundesgerichts. Die erfolgte Volksabstimmung sei aufgehoben worden und nun erneut anzusetzen. Dies ergebe sich einerseits aus den formellen und materiellen Voraussetzungen von Art.139 BV, welche erfüllt seien, und andererseits

22 CORSIN BISAZ, Direktdemokratische Instrumente als «Anträge aus dem Volk an das Volk»: Eine Systematik des direktdemokratischen Verfahrensrechts in der Schweiz, Zürich 2020, N77.

23 Zum schutzwürdigen Interesse an einer Feststellungsverfügung im Allgemeinen etwa PIERRE TSCHANNEN / ULRICH ZIMMERLI / MARKUS MÜLLER, Allgemeines Verwaltungsrecht, 4. Aufl., Bern 2014, §28 $\mathrm{N} 63 \mathrm{f}$.

24 Vgl. TSCHANNEN/ZIMMERLI/MÜLLER (Fn. 23), §28 N64.

25 Vgl. zu den praktischen Folgen dieser Rechtslage unten Rz. $29 f$. 
aus Sinn und Zweck des vorangehenden Urteils des Bundesgerichts. Die Abstimmung sei aufgehoben worden, um das Vertrauen der Stimmberechtigten in den demokratischen Prozess wiederherzustellen, was voraussetze, dass die Abstimmung möglichst unmittelbar wiederholt werde. Es verstosse gegen Treu und Glauben, wenn das Initiativkomitee vor Bundesgericht eine neue Abstimmung erstreite, nur um die Initiative dann zurückzuziehen. Rechtlich verorten die Beschwerdeführer diese Argumente in der Bestimmung von Art. 73 Abs. 2 BPR, wonach ein Initiativkomitee seine Initiative zurückziehen kann, bis der Bundesrat die Volksabstimmung ansetzt. Dies sei mit der ersten Volksabstimmung geschehen. Das Bundesgericht habe diese Abstimmung aufgehoben, womit das Rückzugsrecht aber nicht erneut aufgelebt sei.

\section{Standpunkt der Bundesbehörden}

13 Der Bundeskanzlei kam die Aufgabe zu, in diesem Verfahren den Standpunkt der Bundesbehörden darzulegen. Bundesrat, Bundesversammlung und Bundeskanzlei hatten sich über das Vorgehen nach der Aufhebung der Volksabstimmung durch das Bundesgericht offensichtlich bereits abgestimmt. ${ }^{26}$

14 Die Bundeskanzlei verwies in ihren historischen Ausführungen auf das weitgehende Rückzugsrecht, welches der Gesetzgeber vorsehen wollte. ${ }^{27}$ Dieses Rückzugsrecht wurde bereits anlässlich der ersten Volksinitiative auf Bundesebene relevant, bei welcher der Gesetzgeber dieser Initiative einen Gegenvorschlag gegenübergestellte. ${ }^{28}$ Erst im Jahre 1950 kodifizierte der Bundesgesetzgeber jedoch dieses bereits zuvor auf Unterschriftenbogen vorgesehene Rückzugsrecht. ${ }^{29}$ Während das Initiativkomitee hierbei noch frei war, ob es eine Rückzugsklausel auf den Unterschriftenbogen vorsehen wollte, so erklärte der Gesetzgeber dies 1978 schliesslich für obligatorisch. ${ }^{30}$

15 Für die Bundeskanzlei bestand der Zweck dieser Regelung darin, dem Initiativkomitee zu ermöglichen, aufgeänderte rechtliche oder politische Bedingungen reagieren zu können und damit Abstimmungsleerläufe verhin-

\footnotetext{
26 Vgl. dazu Urteil des Bundesgerichts 1C_105/2020, 1C_129/2020 vom 7. Oktober 2020, Sachverhalt A.

27 Urteil des Bundesgerichts 1C_105/2020, 1C_129/2020 vom 7. Oktober 2020 E.3.2.

28 Botschaft des Bundesrates vom 30. März 1907 an die Bundesversammlung betreffend die Bundesgesetzgebung über die Wasserkräfte (BBl1907 II 624 ff.), S. 633 .

29 Art. 1 Abs. 2 Ziff. 2 Bundesgesetz betreffend die Abänderung des Bundesgesetzes über das Verfahren bei Volksbegehren und Abstimmungen betreffend die Revision der Bundesverfassung vom 5. Oktober 1950, AS $195117 \mathrm{ff}$.

30 Art. 68 lit.c und 73 Abs. 1 BPR, AS 1978699 ff.
}

dern zu können. ${ }^{31}$ Diese Ausführungen treffen ohne Weiteres zu, doch ist zu ergänzen, dass das Rückzugsrecht vor allem auch in Zusammenhang mit dem Institut des Gegenvorschlags zu sehen ist und in dieser Kombination das äusserst erfolgreiche Verhandlungssystem zwischen Initiativkomitee und Gesetzgeber erst ermöglicht. ${ }^{32}$

Diesen Aspekt des Rückzugsrechts erwähnen weder die 16 Bundeskanzlei noch das Bundesgericht. Der Grund mag darin liegen, dass die CVP nach der Aufhebung der Initiative sehr wohl versucht hatte, den Gesetzgeber erneut zu einem Gegenvorschlag zu motivieren. Der Bundesrat verabschiedete denn auch am 14. August 2018 eine Zusatzbotschaft zur bereits laufenden Änderung des Bundesgesetzes über die direkte Bundessteuer (Ausgewogene Paar- und Familienbesteuerung), ${ }^{33}$ welche Anliegen der Initiative aufnehmen sollte. ${ }^{34}$ Die Bundesversammlung wies die Vorlage jedoch im Dezember 2019 an den Bundesrat zurück, ${ }^{35}$ worauf das Initiativkomitee seine Volksinitiative zurückzog. Wenngleich das Anliegen der ausgewogenen Paar- und Familienbesteuerung grosse Sympathien beim Stimmvolk genoss, konnte das Parlament darauf hoffen, dass die CVP ihre Initiative aufgrund der umstrittenen Legaldefinition der Ehe ohnehin zurückziehen würde.

Auch die Bundeskanzlei blendete den Zusammenhang 17 zwischen Rückzugsrecht und Gegenvorschlag aus, als sie auf die Systematik des BPR verwies, wo die Bestimmung über den Rückzug einer Volksinitiative (Art. 73 BPR) unmittelbar nach der Bestimmung über das Zustandekommen einer Volksinitiative folge (Art. 72 BPR) und nicht nach derjenigen über die Behandlung einer Initiative durch das Parlament (Art.75 BPR). Diese Argumentation überzeugt im konkreten Fall wenig, denn der frühestmögliche Zeitpunkt für einen Rückzug einer Volksinitiative ist derjenige nach dem Zustandekommen der Initiative. Art. 73 Abs. 2 BPR erwähnt denn auch bereits den spätestmöglichen Zeitpunkt des Rückzugs: Festsetzen der Volksabstimmung durch den Bundesrat. Dieser Akt findet erst nach Beendigung des parlamentarischen Verfahrens statt, was klar macht, dass der Rückzug während dieser ganzen Zeitspanne möglich ist. In diesem

31 Urteil des Bundesgerichts 1C_105/2020, 1C_129/2020 vom 7. Oktober 2020 E.3.2.

32 Vgl. dazu NICOLAS VON ARX, Ähnlich, aber anders: Die Volksinitiative in Kalifornien und in der Schweiz, Genf 2002, S. 69f.; GABRIELA ROHNER, Die Wirksamkeit von Volksinitiativen im Bund 1848-2010, Zürich 2012, S. $256 \mathrm{f}$.

33 Vgl. Botschaft vom 21. März 2018 zur Änderung des Bundesgesetzes über die direkte Bundessteuer (Ausgewogene Paar- und Familienbesteuerung) (BBl 2018 2133).

34 Zusatzbotschaft zur Änderung des Bundesgesetzes über die direkte Bundessteuer (Ausgewogene Paar- und Familienbesteuerung) (BBl 2019 5787).

35 AB2019S704; AB2019 N2337. 
Sinne wiederholte das Parlament die Beratung über die Initiative zwar nicht förmlich, beschäftigte sich aber sehr wohl inhaltlich noch einmal mit dem Anliegen der Initiative, ${ }^{36}$ was durchaus Anlass für den Rückzug der Initiative hätte bieten können.

In der ganzen Zeitspanne von Zustandekommen bis zur Festsetzung der Volksabstimmung durch den Bundesrat kann das Initiativkomitee aus mannigfaltigen und durchaus rein politischen Erwägungen zum Schluss kommen, seine Initiative zurückzuziehen. Dass das Initiativkomitee nach langen Jahren, in welchen seine Initiative von den Behörden behandelt, vom Stimmvolk bereits einmal abgelehnt und nun womöglich erneut ohne Gegenvorschlag ein zweites Mal zur Abstimmung gekommen wäre, von der Initiative Abstand nehmen will, ist völlig nachvollziehbar und vom eigentlichen Zweck des Rückzugsrechts durchaus erfasst.

\section{Urteil des Bundesgerichts}

Das Bundesgericht teilt die Meinung der Bundeskanzlei, wonach die Rechtsstreitigkeit es erfordert, Art. 73 BPR daraufhin auszulegen, ob das Rückzugsrecht erneut auflebt, nachdem eine Volksabstimmung aufgehoben wurde. ${ }^{37} \mathrm{Zu}$ beantworten ist mit anderen Worten die Frage, ob die Initiative erneut zur Disposition des Initiativkomitees steht oder in der objektiv-rechtlichen Sphäre des Staates verbleiben soll. In der Lehre wird der Moment, in welchem das Rückzugsrecht erlöscht, denn auch - etwas allzu apodiktisch ${ }^{38}$ - als der Moment bezeichnet, in welchem das Initiativkomitee die Herrschaft über «seine» Volksinitiative verliere. ${ }^{39}$ Dieser Übergang der Initiative in die Sphäre des Staates hängt mit der Doppelnatur der politischen Rechte zusammen, welche Individualrechte sind, denen aber - in der Schweiz meist gleichzeitig - auch öffentliche Funktionen zukommen (dazu unten Rz. 32 ff.). ${ }^{40}$

36 Vgl. Urteil des Bundesgerichts 1C_105/2020, 1C_129/2020 vom 7. Oktober 2020 E.3.3.

37 Urteil des Bundesgerichts 1C_105/2020,1C_129/2020 vom 7. Oktober 2020 E.3.4.

38 Initiativkomitees nehmen mitunter Einfluss auf die Ausführungs gesetzgebung, sei dies durch parlamentarischen Einfluss, durch Einsitznahme in Arbeitsgruppen oder durch die Drohung, ein Referendum gegen die Ausführungsgesetzgebung zu ergreifen. Vgl. dazu GORAN SEFEROVIC, Volksinitiative zwischen Recht und Politik: Die staatsrechtliche Praxis in der Schweiz, den USA und Deutschland, Bern 2018, N166.

39 CORSIN BISAZ, Das Initiativkomitee, in: Good/Platipodis (Hrsg.) Direkte Demokratie: Herausforderungen zwischen Recht und Politik: Festschrift für Andreas Auer zum 65. Geburtstag, Bern 2013, S.141.

40 Vgl. BISAZ, Direktdemokratische Instrumente (Fn. 22), N77 ff.; zur Doppelnatur der politischen Rechte im Allgemeinen auch Ders., N 58 ff.; PIERRE TSCHANNEN, Stimmrecht und politische Verständigung: Beiträge zu einem erneuerten Verständnis von direkter Demokratie, Basel 1995, N20ff.
Das Bundesgericht geht diese Frage chronologisch an, 20 weshalb es sich zuerst dem Bundesbeschluss über die Volksinitiative aus dem Jahre 2015 widmet. ${ }^{41}$ Dieser Beschluss, mit welchem die Bundesversammlung die Initiative gültig erklärte und zur Ablehnung empfahl, behalte seine Gültigkeit. ${ }^{42}$ Dies trifft $\mathrm{zu}$, die Bundesversammlung hätte es aber in der Hand gehabt, auf ihren damaligen Beschluss zumindest faktisch zurückzukommen. Der Bundesrat wollte die neue Abstimmung nämlich nicht ansetzen, bevor das Parlament sich über das Anliegen der Initiative eine aktualisierte «Gesamtbetrachtung» verschafft hatte. ${ }^{43}$ Damit räumte er der Bundesversammlung die Möglichkeit ein, einen Gegenvorschlag zu beschliessen. Den Bundesbeschluss über die Volksinitiative zog der Bundesrat hingegen nicht in Wiedererwägung.

Nach Art. 73 Abs. 2 BPR beendet die Ansetzung der Volks- 21 abstimmung durch den Bundesrat die Frist, innerhalb welcher das Initiativkomitee seine Volksinitiative zurückziehen kann. Der Wortlaut der Bestimmung äussert sich aber nicht zur Frage, ob dies auch gilt, wenn eine zweite Volksabstimmung über eine Initiative angesetzt wird. Die Bestimmung ist somit auslegungsbedürftig.

Zu Recht folgt das Bundesgericht nicht der Argumenta- 22 tion der Bundeskanzlei, wonach aus der systematischen Stellung des Rückzugsrechts im BPR für den Fall des Rückzugs nach Aufhebung einer Volksinitiative etwas abzuleiten sei. ${ }^{44}$

Ebenso wenig folgt das Gericht aber den Beschwerde- 23 führern und sieht es nicht durch Sinn und Zweck der ersten, in BGE145 I 207 und Urteil 1C_315/2018 gutgeheissenen Beschwerden für geboten, die Abstimmung obligatorisch zu wiederholen. Die Abstimmungsfreiheit von Art. 34 Abs. 2 BV sei bereits mit diesen Urteilen Nachachtung verschafft worden. Das Wiederaufleben des Rückzugsrechts stehe der Abstimmungsfreiheit nicht entgegen. ${ }^{45}$

Nach Ansicht des Bundesgerichts wollte der historische 24 Gesetzgeber das Rückzugsrecht möglichst erleichtern und die Dispositionsfreiheit des Initiativkomitees stärken. Das Rückzugsrecht werde nur deshalb zeitlich auf

41 Bundesbeschluss vom 19. Juni 2015 über die Volksinitiative «Für Ehe und Familie - gegen die Heiratsstrafe» (BBl 20154849).

42 Urteil des Bundesgerichts 1C 105/2020, 1C 129/2020 vom 7. Oktober 2020 E.3.6.

43 Zusatzbotschaft zur Änderung des Bundesgesetzes über die direkte Bundessteuer (Ausgewogene Paar- und Familienbesteuerung) (Fn.34), S. 5794 .

44 Vgl. dazu auch schon oben Rz.17.

45 Urteil des Bundesgerichts 1C 105/2020,1C 129/2020 vom 7. Oktober 2020 E.3.6 (S.13). 
den Zeitpunkt der Ansetzung der Volksabstimmung beschränkt, weil Behörden und Stimmberechtigte sonst bis kurz vor dem Abstimmungstermin nicht wüssten, ob die Abstimmung stattfinde. ${ }^{46}$ Der Normzweck - eine möglichst weitgehende Dispositionsfreiheit des Initiativkomitees in Bezug auf das Rückzugsrecht - stehe einem Rückzugsrecht im Vorfeld einer erneuten Abstimmung nicht entgegen. ${ }^{47}$

Dem ist aus heutiger Sicht zuzustimmen, doch ist aus historischer Warte anzufügen, dass die Bundesbehörden das Rückzugsrecht einst vor allem deshalb begrüssten, weil sie eine Reihe von Volksinitiativen seit der Zeit des Ersten und bis nach Ende des Zweiten Weltkrieges verschleppten oder schlicht nicht behandelten. Das Rückzugsrecht ermöglichte es den Behörden, die Initiativkomitees dazu anzuhalten, die inzwischen nicht mehr aktuellen Initiativen zurückzuziehen. ${ }^{48}$

Das Argument der Beschwerdeführer, es widerspreche Treu und Glauben, zuerst die Aufhebung der Volksabstimmung zu erwirken, nur um die Initiative danach zurückzuziehen, entkräftet das Bundesgericht mit dem Hinweis, wonach das Rückzugsrecht in der alleinigen Disposition des Initiativkomitees stehe und dieses seinen Entscheid nicht zu begründen brauche.

27 Zusammenfassend kommt das Gericht zum Schluss, es gebe keine stichhaltigen Gründe, Art. 73 Abs. 2 BPR einschränkend auszulegen. Im Gegenteil sei dem Initiativkomitee der Rückzug ihrer Initiative, auch nachdem die ersteVolksabstimmungaufgehobenwurde, zugestatten. ${ }^{49}$

\section{Schlussfolgerungen} gerichts, indem es Art.73 BPR weitgehend gestützt auf das historische und teleologische Auslegungselement im Sinne einer weitgehenden Dispositionsfreiheit des Initiativkomitees auslegt. In prozessualer Hinsicht ist $\mathrm{zu}$ begrüssen, dass das Bundesgericht gestützt auf Art. 34 Abs. 2 BV i.V.m. Art. 29a BV eine Beschwerde in Stimmrechtssachen bereits gegen den Rückzugsentscheid des

46 Urteil des Bundesgerichts 1C_105/2020,1C_129/2020 vom 7. Oktober 2020 E. 3.6 (S.13).

47 Urteil des Bundesgerichts 1 C_105/2020, 1C_129/2020 vom 7. Oktober 2020 E.3.6 (S.13).

48 Vgl. ANDREAS KLEY, Volksinitiativen: Das Parlament als Vermittler zwischen Volk, Regierung und Gerichten?, Parlament: Mitteilungsblatt der Schweizerischen Gesellschaft für Parlamentsfragen 18, 2015, S. 40; ANDREAS KLEY, Von Stampa nach Zürich: Der Staatsrechtler Zaccaria Giacometti, sein Leben und Werk und seine Bergeller Künstlerfamilie, Zürich 2014, S. 281f.

49 Urteildes Bundesgerichts 1C_105/2020,1C_129/2020 vom 7.Oktober 2020 E. 3.7.
Initiativkomitees zulässt. Dies insoweit, als eine solche Beschwerde nach Art. 29a Satz 2 BV nicht gesetzlich ausdrücklich ausgeschlossen und diese Ausnahme durch spezifische, qualifizierte Gründe gerechtfertigt ist. ${ }^{50}$ Das Urteil wirft aber andererseits auch eine Reihe von prozessualen Fragen auf.

\section{Verfassungskonforme Auslegung von Art. 80 Abs. 2 und 3 BPR}

Das Bundesgericht scheint bemüht, die Singularität des 29 Falles zu betonen, wenn es Art. 80 Abs. 2 und 3 BPR lediglich angesichts «beachtlicher Gründe» und «in der vorliegenden Konstellation» analog anwenden will. Die präjudizielle Bedeutung wird weiter abgeschwächt, indem das Gericht ausdrücklich offenlässt, ob Anfechtungsobjekt der Rückzugsentscheid des Initiativkomitees oder die allfällige Verfügung der Bundeskanzlei bilden soll. Wenngleich die Rückzugserklärung selbst das eigentliche Anfechtungsobjekt bildet, ${ }^{51}$ wird der Rückzugsentscheid in der Praxis wohl regelmässig erst mit der Publikation durch die Bundeskanzlei publik werden, womit sich die Unklarheit prozessual wenig auswirken dürfte.

Da die Beschwerde in Stimmrechtssachen aber nicht an 30 spezifische Anfechtungsobjekte anknüpft, sondern sich gegen eine Vielzahl unterschiedlicher Handlungen von Behörden und Privater richten kann, ist das Anfechtungsobjekt andererseits aber auch nicht entscheidwesentlich.52 Mit diesen Besonderheiten der Beschwerde in Stimmrechtssachen sollte eine Beschwerde gegen einen Rückzugsentscheid des Initiativkomitees denn auch ohne ausdrückliche gesetzliche Erwähnung zulässig sein.

Die erste öffentlich-rechtliche Abteilung des Bundesge- 31 richts schafft mit dem vorliegenden Urteil auch einen gewissen Gegensatz zu BGE146 I126. In jenem Urteil verneinte die gleiche Abteilung die Anfechtbarkeit der Verfügung der Bundeskanzlei über das Zustandekommen eines Referendums. Das Gericht stützte sich dafür auf den Wortlaut von Art. 80 Abs. 2 BPR, wonach gegen Verfügungen der Bundeskanzlei über das Nicht-Zustandekommen eines Referendums die Beschwerde an das

50 In diesem Sinne auch GEROLD STEINMANN, Bundesgericht, I. öffentlich-rechtliche Abteilung, 24. März 2020,1C_134/2020 = BGE146I126 ZBl 2020, S. 515f. Vgl. allg. zu gesetzlichen Ausnahmen der Rechtsweggarantie ANDREAS KLEY, Kommentar zu Art. 29a BV N20, in: Ehrenzeller/Schindler/Schweizer/Vallender (Hrsg.), Die schweizerische Bundesverfassung, St. Galler Kommentar, 3. Aufl., Zürich 2014; BERNHARD WALDMANN, Kommentar zu Art. 29a BV N 22, in: Waldmann/Belser/Epiney (Hrsg.), Basler Kommentar BV, Basel 2014.

51 Siehe oben Rz.9f.

52 GEROLD STEINMANN / ADRIAN MATTLE, Kommentar zu Art. 82 BGG N78, 85 ff., in: Niggli/Uebersax/Wiprächtiger/Kneubühler (Hrsg.), Basler Kommentar Bundesgerichtsgesetz, 3. Aufl., Basel 2018 (zit. BSK BGG-BEARBEITERIN). 
Bundesgericht zulässig ist. Im Umkehrschluss sei daher die Beschwerde gegen die Verfügung über das Zustandekommen eines Referendums kein taugliches Anfechtungsobjekt. ${ }^{53}$ In Anbetracht der in der Lehre geäusserten Kritik an diesem Entscheid ${ }^{54}$ wäre zu überlegen, ob der aus Art. 80 Abs. 2 BPR abgeleitete e contrario-Schluss tatsächlich eine hinreichend klare gesetzliche Grundlage für eine Ausnahme von der Rechtsweggarantie nach Art. 29a Satz 2 BV bilden kann. Im vorliegenden Urteil kam das Gericht hingegen zum Schluss, dass das BPR den ersuchten Rechtsschutz gerade nicht ausschliesse, weshalb dieses verfassungskonform «auszulegen und anzuwenden» sei. ${ }^{55}$ Art. 80 Abs. 2 und 3 BPR erwähnen mit keinem Wort die Verfügungen der Bundeskanzlei über den Rückzug einer Volksinitiative, womit das Bundesgericht nach seinen Erwägungen in BGE146 I126 die Anfechtbarkeit durchaus hätte verneinen können.

\section{Beschwerde in Stimmrechtssachen und duale Natur der politischen Rechte} zu. Diese sind sowohl gerichtlich durchsetzbares Individualrecht als auch Grundlage für die Willensbildung des Volks als Staatsorgan, welches sich aus den einzelnen Stimmberechtigten zusammensetzt. ${ }^{56}$ In der Schweiz überwiegt dabei die Auffassung, den politischen Rechten und damit auch der Beschwerde in Stimmrechtssachen kämen meist beide Funktionen gleichzeitig zu. ${ }^{57}$ So können Stimmberechtigte mit der Beschwerde in Stimmrechtssachen sowohl persönliche als auch öffentliche Interessen wahrnehmen. ${ }^{58}$

Dass Lehre und Rechtsprechung heute wenig zwischen diesen beiden Funktionen differenzieren, ${ }^{59}$ zeigt sich etwa daran, dass die Rechtsnatur eines Initiativkomitees wenig geklärt ist. ${ }^{60}$ Dieses steht an der Schnittstelle zwischen engagierten aber unorganisierten Stimmberechtigten, die sich zum Initiativkomitee zusammenschliessen, welches dannzumal eine Volksinitiative zustande bringen kann. Das Initiativkomitee wird denn auch als

\section{BGE146I126 E.1.3.}

54 LUKA MARKIĆ, Von Verfügungen über das (Nicht-) Zustandekommen eidgenössischer Referenden und den Tücken bei deren Anfechtung, sui generis 2020, S. 395 ff., N15ff.; STEINMANN (Fn. 50), S. 509 ff.; PIERRE TSCHANNEN, Die staatsrechtliche Rechtsprechung des Bundesgerichts in den Jahren 2019 und 2020, VII. Andere verfassungsmässige Rechte, ZBJV 2020, S. $574 \mathrm{ff}$.

55 Urteil des Bundesgerichts 1C_105/2020,1C_129/2020 vom 7.Oktober 2020 E. 2.3.

56 Vgl. schon oben Rz.19.

57 Vgl. für die Beschwerde in Stimmrechtssachen BSK BGG STEINMANN/MATTLE, Art. 89 N 72.

58 BSK BGG-STEINMANN/MATTLE, Art. 89 N 72.

59 BISAZ, Direktdemokratische Instrumente (Fn. 22), N59.

60 Vgl. BISAZ, Initiativkomitee (Fn. 39), S.135ff. «intermediäres Staatsorgan» ${ }^{\boldsymbol{1}}$ bezeichnet. Dieser Begrifflässt es offen, welche Handlungen des Initiativkomitees bereits als staatsnahe Handlungen aufgefasst werden können und damit grundsätzlich Anfechtungsobjekt einer Beschwerde in Stimmrechtssachen bilden können.

In Deutschland, wo die duale Natur der politischen Rech- 34 te in der Gerichtspraxis eine grössere Bedeutung einnimmt, ist äusserst umstritten, ab welchem Zeitpunkt ein Initiativkomitee staatliche Funktionen wahrnimmt. ${ }^{62}$ Immerhin sind sich Lehre und Bundesverfassungsgericht einig, dass ein zustande gekommenes Volksbegehren staatliche Organpositionen begründet und das Initiativkomitee die Herrschaft über «seine» Initiative damit grundsätzlich verliert. ${ }^{63}$

In der Schweiz soll das Initiativkomitee sein Rückzugs- 35 recht nach dem Willen des Gesetzgebers möglichst frei ausüben können und braucht daher einen Rückzug nicht zu begründen. Gleichwohl hat es das Initiativkomitee damit in der Hand, den politischen Willen von 100’000 Stimmberechtigten annullieren zu können, welche die Initiative unterzeichnet haben. ${ }^{64}$ Das Initiativkomitee nimmt damit zumindest nicht mehr nur eigene Rechte wahr, womit der Rückzug einer Volksinitiative auch die politischen Rechte der Stimmberechtigten als Unterzeichnerinnen und Unterzeichner der Volksinitiative betrifft. Gleichwohl sieht das Gesetz vor, dass der Entscheid über den Rückzug bei der Mehrheit des Initiativkomitees liegt, womit für eine Verfügung der Bundeskanzlei kein Raum bleiben kann. Im Hinblick auf die Praxis der Stimmrechtsbeschwerde wäre es zu wünschen gewesen, dass das Bundesgericht dies klargestellt hätte, zumal dies auch der Auffassung der Bundeskanzlei zu entsprechen scheint. 